Jörg Klein

\title{
Die biologischen Wurzeln des Inzestverbots
}

\begin{abstract}
Does an inclination towards incest exist and why is incest prohibited? There are mainly two points of view: that of Sigmund Freud, the founder of psychoanalysis, and that of Edward Westermarck, a Finnish-British anthropologist. Freud is of the opinion that men have a profound desire for incest, whereas Westermarck presumes an instinctive aversion against it. Nowadays the discussion has received fresh impulse due to a modern interpretation of Westermarck's theory and the controversy over the sexual abuse of children in their families. The author is in favour of the Westermarck theory and tries to explain the prohibition of incest based on a natural inhibition towards incest.
\end{abstract}

\section{Gelehrtendisput}

Schon lange Zeit bevor der Inzest zu einem prominenten Thema der Soziobiologie und, hiervon unabhängig, im Zusammenhang mit sexuellem Mißbrauch Thema allgemeinen öffentlichen Interesses wurde, fand ein Gelehrtenstreit unter Anthropologen, Ethnologen, Psychologen und Soziologen statt. Obwohl er mit großem Engagement ausgetragen wurde, erlangte er über einen relativ kleinen Kreis von Fachwissenschaftlern hinaus kaum Beachtung. Der Disput hatte zum Thema, warum Menschen normalerweise keinen Inzest praktizieren, warum diejenigen Personen, zu denen quantitativ wie qualitativ engster Kontakt gepflegt wird, die eigenen Eltern, Geschwister und Kinder, als Geschlechts- und Ehepartner ausgeschlossen sind.

Diese erste Phase der Inzestdiskussion setzte gegen Ende des 19. Jahrhunderts ein und dauerte bis etwa zur Mitte unseres Jahrhunderts. Ihre stärkste Prägung erhielt sie durch die einander diametral entgegengesetzten Positionen des in England wirkenden finnischen Anthropologen Edward Westermarck und des Begründers der Psychoanalyse Sigmund Freud.

1891 stellte Westermarck in der ersten Auflage seines Werkes The History of Human Marriage die These auf, daß ein angeborener Widerwille gegen den geschlechtlichen Verkehr zwischen Personen existiere, die von früher Jugend an eng beisammen gelebt haben. (Genauer sprach er später vom Widerwillen zwischen Personen, die entweder von der Kindheit einer von ihnen oder beider an zusammen gelebt haben.) Der Mechanismus betreffe nahe Blutsverwandte zwar nicht in jedem Fall und ausschließlich, aber doch im Normalfall und während der längsten Zeit der menschlichen Geschichte. Er diene der Vermeidung erbkranken 
Nachwuchses und habe sich im Verlauf der biologischen Evolution herausgebildet.

Freud bezog seine Position erstmals 1900 in seinem Werk Die Traumdeutung und, explizit in Gegnerschaft zu Westermarck, 1912/13 in Totem und Tabu. Er behauptete für den Menschen das Vorhandensein starker, wenn auch zumeist unbewußter Inzestwünsche, deren Bezähmung durch väterlichen Eingriff hauptsächlich beim kleinen Kinde in der 'ödipalen Phase' und später erneut in der Pubertät erfolge. Der unbewußte Inzestwunsch scheint nach Freud jedoch auch beim Erwachsenen nie völlig zu erlöschen.

Obwohl beide Kontrahenten ihre Gedanken zum Inzest über Jahrzehnte wiederholten, ausbauten und weiterverfochten, traf allein Freud in der zeitgenössischen Wissenschaftswelt auf Unterstützung. Eine Ausnahme bildete einzig der bedeutende englische Sexualwissenschaftler Havelock Ellis (1906; 1932), der sich der Position Westermarcks anschloß. Auch Westermarcks Kollegen aus der Anthropologie und Ethnologie akzeptierten die Freudsche Position und nahmen sie als Prämisse für ihre neuen Theorien zum Inzestverbot. Mit diesen Theorien entstanden dann, grundsätzlich dem Freud-Lager verhaftet, verschiedene weitere Gruppierungen in der Inzestdiskussion.

Freud hatte sich überwiegend mit dem starken Inzestbegehren und den es unterdrückenden psychologischen Mechanismen befaßt. Die für die Ethnologen zentrale Frage war dagegen, warum diesem Begehren eigentlich ein Verbot entgegensteht.

Hier vertraten Bronislaw Malinowski $(1931 ; 1979 ; 1981)$ und Brenda $Z$. Seligman $(1929 ; 1932 ; 1935 ; 1950)$ die Auffassung, daß im Falle erlaubten und dann regelmäßig ausgeübten Inzests alle Familien von Eifersucht und einer völligen Verwirrung ihrer familialen Rollen - als Elter, Kind, Geschwister - heimgesucht, dadurch ins Chaos gestürzt und zerrissen würden. Die Familie aber sei die Basiseinheit der Gesellschaft, unverzichtbar als Erziehungsinstitution und für die Bereitstellung von Vorbildern. Ohne Inzestverbot gäbe es damit weder Familie noch Gesellschaft, was wiederum hieße, daß beide Institutionen auch erst mit Errichtung des Verbots in menschlicher Frühzeit entstanden wären.

Eine vom Inzest ausgehende existentielle Bedrohung nur für die Gesellschaft sahen die Vertreter der sogenannten Allianztheorie: Reo Fortune (1932), Leslie A. White (1948) und Claude Lévi-Strauss (1984). Die Familien selbst kämen mit dem Inzest wohl zurecht, würden sich aber, als Inzestfamilien am Ziel ihrer Wünsche, von jeder sozialen Außenwelt abschließen und die Existenz 'isolierter Monaden' führen. Erst der vom Inzestverbot bewirkte Zwang zur Ausheirat stelle soziale Kontakte und Bindungen, 'Allianzen', zwischen den Familien her. Wieder habe Gesellschaft, hier als auf dem Fundament zwischenfamilialer Kontakte ruhend aufgefaßt, erst mit Errichtung des Verbots in menschlicher Frühzeit begonnen.

Wieder andere, an erster Stelle George Peter Murdock (1949), versuchten zwischen den Inzestverbotsschulen zu vermitteln und rechneten dem Verbot sowohl die beschriebene 'intra-' als auch die 'interfamiliale' Funktion zu (wobei offensichtlich der Widerspruch übersehen wurde, daß im Falle der Inzestfreigabe 
die Familien schwerlich zerrissen werden und zugleich sich isoliéren würden). Der Soziologe Talcott Parsons (1954) wiederum glaubte, daß die Kinder nicht zu Erwachsenenpersönlichkeiten heranreifen könnten, wenn sie, ihre Inzestwünsche realisierend, keine Ablösung von den Eltern vornähmen. Der Gesellschaft fehlten damit handlungsfăhige Erwachsene zur Übernahme sozialer, ökonomischer und religiöser Funktionen.

Erbhygienische Funktionen für das Verbot wurden in der soziologisch-ethnologischen Freudschule explizit zurückgewiesen, und sexueller Mißbrauch war im Zusammenhang mit Inzest überhaupt noch kein Thema.

\section{Belege für den starken Inzestwunsch des Menschen}

Für die menschliche Inzestneigung wurden und werden hauptsächlich vier Belege vorgebracht: die Tatsache des Verbots (1), das Vorkommen des Inzests (2), der Inhalt von Schlafträumen (3) und von poetischen Fiktionen (4).

(1) Die Tatsache des universal vorkommenden strengen Inzestverbots verweise bereits zur Genüge auf das Vorhandensein verbreiteter und starker Inzestbedürfnisse (Frazer 1910; Freud 1974; White 1948; Lévi-Strauss 1984; Aberle et al. 1963). So heißt es z. B. bei White $(1948,416)$ :

"But if this (ein angeborener Widerwille gegen sexuelle Verbindungen mit nahen Verwandten, $d$. Verf.) were the case, why should societies enact strict laws to prevent them? Do not, as a matter of fact, the stringent and worldwide prohibitions indicate a universal and powerful desire for sexual unions with one's relatives?"

Hiergegen ist einzuwenden, daß das Inzestverbot vermutlich nicht universal ist (Klein 1991, 99-117) und daß die mit ihm verbundenen Sanktionen von Kultur zu Kultur teilweise stark variieren (bezüglich des Geschwisterinzests: Fox 1962). Die innere Logik des Belegs wird auch dadurch erschüttert, daß strenge Normen sich durchaus gegen einen nur kleinen Kreis potentieller Täter richten können, nämlich dann, wenn die von ihnen ausgehende reale oder vermeintliche Bedrohung groß genug ist.

(2) $\mathrm{Zu}$ einer Realisierung der starken Inzestwünsche soll es, als Folge des mächtigen Inzesttabus, nur selten kommen. Doch auch schon der seltene Inzest demonstriere, daß es eine instinktive Inzesthemmung nicht geben könne. Bedenke man nun die große Nähe zwischen Eltern und Kindern und zwischen Geschwistern und ihre prinzipielle gegenseitige Verfügbarkeit als Sexualpartner, müsse auf ein allgemein verbreitetes Inzestverlangen geschlossen werden (z.B. Seligman 1935; Lévi-Strauss 1949).

Gegen diesen Schluß von seltenen Inzestfällen auf ein allgemein verbreitetes Inzestverlangen lassen sich mehrere Kritikargumente anführen. Erstens müssen Instinkte, um ihrer Funktion gerecht zu werden, nicht unfehlbar sein. Sporadisch vorkommender Inzest ist mit der These, daß eine erblich fixierte Inzestscheu existiert, durchaus vereinbar. Zweitens könnten auf der Basis einer solchen Argumentation alle möglichen selten gezeigten Verhaltensformen - jedenfalls 
dann, wenn prinzipiell Gelegenheiten für sie vorhanden sind - so gedeutet werden, daß ein allgemeines und starkes, wenn auch irgendwie unterdrücktes Verlangen nach ihrer Praktizierung vorläge. Woran sollten dann aber wirklich selten erlebte Bedürfnisse überhaupt erkennbar sein? Zuletzt stellt sich die Frage, warum einem gegen stärkste sexuelle Leidenschaft antretenden Inzestverbot ein derart durchschlagender Erfolg beschieden sein soll. Verbote zum Beispiel der Homosexualität, des vor- und außerehelichen Geschlechtsverkehrs oder der Onanie haben es kaum je vermocht, die betreffenden Verhaltensweisen zur Rarität werden zu lassen.

(3) Häufig werden als Beweis für die menschliche Inzestneigung Erfahrungen aus psychoanalytischen Behandlungen herangezogen, und hier an erster Stelle die Schlafträume der Patienten. Erstaunlicherweise kommt aber nach Therapeutenwie Patientenberichten in den meisten Träumen, die hier als Inzestträume gelten, Inzest gar nicht vor. Vielmehr zeigt dieser sich erst im Prozeß der psychoanalytischen Traumdeutung als Symbolgehalt von einem äußerlich diesbezüglich ganz unverfänglichen Traumgeschehen. Hierzu Freud selbst $(1972,389)$ :

"Wenn ich gegen Patienten die Häufigkeit des Ödipustraumes, mit der eigenen Mutter geschlechtlich zu verkehren, betone, so bekomme ich zur Antwort: Ich kann mich an einen solchen Traum nicht erinnern. Gleich darauf steigt aber die Erinnnerung an einen anderen, unkenntlichen und indifferenten Traum auf, der sich bei dem Betreffenden häufig wiederholt hat, und die Analyse zeigt, daß dies ein Traum des gleichen Inhalts, nämlich wiederum ein Ödipustraum ist. Ich kann versichern, daß die verkappten Träume vom Sexualverkehre mit der Mutter um ein Vielfaches häufiger sind als die aufrichtigen."

Wenn jemand behauptet, daß etwas ganz oder hauptsächlich im Versteckten existiere, trägt er eine besondere Beweislast. Nun wäre der Inzestwunsch schon dann als mehr oder weniger verborgen anzusehen, wenn er sich nur in Träumen zeigte. Geht die Behauptung aber dahin, daß er selbst in den Träumen ein nur kryptisches Dasein führe, möchte man hierfür doch überzeugende Argumente hören. Diese werden aber von der psychoanalytischen Traumdeutung nicht geliefert. Aus der Fülle der Literatur sei hier nur auf die ausgezeichnete Kritik an der psychoanalytischen Traumdeutung (und darüber hinaus an der Psychoanalyse überhaupt) von Dieter E. Zimmer $(1984 ; 1986)$ verwiesen.

(4) Als starker Beleg gilt Anhängern der Psychoanalyse, daß Werke von Dichtern, daß Mythen, Märchen, Schauspieldramen und Romane immer wieder Inzestwünsche zum Ausdruck brächten. Doch wie bei den Schlafträumen handeln auch die meisten der hier angeführten poetischen Texte eben nur für den Anhänger der Psychoanalyse von Inzestwünschen. Sie werden - unter Rückgriff auf die Prinzipien der psychoanalytischen Traumdeutung - in den Text hineingedeutet.

Ich selbst habe einige der bekannteren psychoanalytischen Interpretationen poetischer Werke (u.a. König Oidipus, Hamlet, Homo faber) alternativen Deutungen gegenübergestellt, wobei letztere sich, an literaturwissenschaftlichen Maßstäben der Textinterpretation gemessen, als deutlich überlegen erwiesen (Klein 1991, 125-139). 


\section{Belege für Westermarck}

Westermarck selbst vermochte nur darauf hinzuweisen, daß menschliches Inzestverlangen, von extremen Ausnahmefällen abgesehen, nirgends feststellbar sei. Eine stark positive Veränderung der Beweissituation trat dann, Jahrzehnte nach seinem Tod, in den 70er Jahren unseres Jahrhunderts ein.

In den Siedlungen der israelischen Kibbuzim wuchsen bis vor nicht langer Zeit die meisten Kinder nicht im Elternhaus heran, sondern in von Erzieherinnen betreuten separaten Horten von je sechs bis acht in etwa gleichaltriger Jungen und Mädchen. Die gemeinsam Aufgewachsenen unterlagen keinem Verbot, als Jugendliche oder Erwachsene sexuelle Beziehungen zueinander aufzunehmen oder später untereinander zu heiraten. Nicht nur, daß Verbote abwesend waren, in Übereinstimmung mit den pädagogischen Zielen der Kibbuz-Bewegung herrschte eine Atmosphäre sexueller Unverkrampftheit. Als Quasi-Geschwister, jedoch keinem Inzestverbot unterworfen, stellten die Kibbuz-Kinder ein 'natürliches', ungeplantes Experiment zur Überprüfung der Westermarck-Theorie dar.

1971 lieferte der israelische Soziologe Joseph Shepher eine gründliche Studie über die Ehepartnerwahl der im Kibbuz Großgewordenen, die fast alle Erwachsenen der zweiten Kibbuz-Generation umfaßte. Zum Ergebnis hatte sie, daß unter 2769 Ehen ehemaliger Kibbuzkinder gerade 13 waren, bei denen die Partner derselben Hortgruppe entstammten. Für die wenigen Ausnahmefälle galt überdies, daß sich hier die Partner entweder erst nach dem sechsten Lebensjahr kennengelernt hatten oder aber während der ersten sechs Jahre jeweils jahrelang getrennt gewesen waren.

Studien über sexuelle Beziehungen von Jugendlichen im Kibbuz, die aus insgesamt fünf Kibbuzim vorliegen (Spiro 1958; Talmon 1964; Shepher 1971) erbrachten ein vergleichbares Ergebnis: Es war nur in solchen Fällen zu Liebschaften gekommen, bei denen die Partner erst im späteren Kindesalter oder als Jugendliche zusammengetroffen waren.

Shepher $(1971 ; 1983)$ interpretiert seine Daten als Beleg für Westermarck. Andere Faktoren als die von Kindheit an erlebte Vertrautheit - so die geringen Partnerwahlmöglichkeiten innerhalb der Erziehungsgruppen - mögen ebenfalls wirksam sein, jedoch ohne daß hierbei eine alternative Erklärung der phänomenal niedrigen Rate vorehelicher Beziehungen und Eheschließungen zwischen den Kindheitskumpanen des Kibbuz sichtbar würde (zur dennoch kontroversen Diskussion siehe: Shepher 1983; Harris 1980; Leavitt 1990; Klein 1991).

Ein weiterer starker Beleg für die Position Westermarcks stammt aus Taiwan. Dort kommt der aussterbende Brauch der 'Kleinen Hochzeit' (Hsiao-hun) vor. Nach einer Kinderverlobung wird die Braut, die sich zu diesem Zeitpunkt noch im Babyalter befindet, von der Familie ihres künftigen Mannes adoptiert und dort aufgezogen. Bald nach der Pubertät des Mädchens wird das wie Bruder und Schwester herangewachsene Paar verheiratet. Bis etwa 1920 kam diese Art von Eheschließung regional ähnlich häufig und weitgehend auch im selben sozialen Milieu vor wie die - mit größerem Aufwand begangene - 'Große Hochzeit' (Ta- 
hun), bei der die Ehepartner eine getrennte Kindheit verbracht hatten und erst am Hochzeitstag selbst zusammengefürt wurden.

In einer Serie ethnologischen Studien ging der Amerikaner Arthur P. Wolf (1966; 1968; 1970; in Zusammenarbeit mit Ch. Huang: 1980) der Frage nach, wie erfolgreich die 'Kleinen Ehen' im Vergleich zu den 'Großen Ehen' waren. Die Durchsicht amtlicher Haushaltsregister ergab, daß in den Kleinen Ehen mehr als ein Viertel weniger Kinder geboren wurden als in den Großen Ehen, offenbar deshalb, weil die Partner der kleinen Ehen seltener zusammen schliefen. Die Scheidungsrate war bei den Kleinen Ehen zweieinhalbmal so hoch wie bei den Großen, und auch bei den außerhelichen Affären, die Wolf (1980) bezüglich der Ehefrauen nach einem ausgeklügelten Plan zu erschließen suchte, lagen sie statistisch deutlich in Führung.

Einen ähnlichen Ehevergleich wie Wolf unternahm die Anthropologin Justine McCabe (1983) im Libanon. Dort werden sehr häufig Ehen zwischen Cousins und Kusinen geschlossen, die entweder in einem gemeinsamen erweiterten Haushalt oder doch im täglichen Kontakt naher Nachbarschaft aufgewachsen sind. Die Scheidungsrate liegt bei diesen Ehen, wie die Forscherin allerdings nur anhand eines einzelnen Dorfes erhob, mehr als viermal so hoch und die Geburtenrate um ein Viertel niedriger als bei den Vergleichsehen, bei denen die Partner nicht zusammen aufgewachsen waren.

\section{Sexueller Kindesmißbrauch und Inzestzahlen}

In den 70er Jahren formierte sich neben der bereits bestehenden Freud- und Westermarck-Schule in den USA noch ein weiterer Standpunkt innerhalb der Inzestdiskussion. Im Zusammenhang mit der Erforschung des sexuellen Kindesmißbrauchs wurde der einzige Konsens, der bis dahin in der Diskussion bestanden hatte, nämlich die Auffassung von der Seltenheit des Inzests, aufgekündigt: Inzest sei vielmehr ein häufig begangenes, ja alltägliches Verbrechen, bei dem zumeist Töchter Opfer physischer und psychischer Gewalt ihrer Väter würden (Armstrong 1978; Justice/Justice 1979; Rush 1982; Gardener Sirtl [Hrsg.] 1983; Kavemann/ Lohstöter 1984; Rijnaartz 1988; Lappessen 1991).

Vor fast zehn Jahren stellten die Autorinnen Barbara Kavemann und Ingrid Lohstöter in ihrem Buch Väter als Täter eine Hochrechnung auf, wonach in der Bundesrepublik pro Jahr 300000 Kinder und Jugendliche bis zum Alter von 18 Jahren, vor allem Mädchen, Opfer sexueller Delikte würden. Die Schätzung erhielt in der Folgezeit allgemeine Publizität und Akzeptanz, wobei sie zumeist nur noch auf Kinder und nicht mehr zusätzlich auf Jugendliche bezogen wurde. Verschiedentlich wurden noch weit höhere Zahlen genannt (z.B. Bild vom 28.8.1990: 1,2 Millionen). Erst in jüngster Zeit mehren sich Stimmen, wonach sexuelle Übergriffe auf Kinder und Jugendliche in Wirklichkeit erheblich seltener sein könnten.

$\mathrm{Zu}$ den noch immer gängigen hohen Mißbrauchszahlen ist anzumerken (vgl. hierzu auch Rutschky 1992): 
(1) Den Straftatbestand des sexuellen Kindesmißbrauchs ( $\$ 176 \mathrm{StGB}$ ) erfüllen eine Vielzahl unterschiedlicher und unterschiedlich zu bewertender Vorfälle: exhibitionistische Akte ebenso wie Vergewaltigungen, einmalige Übergriffe, aber auch jahrelange Viktimisienungen. Der Begriff des sexuellen Kindesmißbrauchs wird aber von verschiedenen Autoren aus der Sozialpädagogik und Sozialarbeit auf einen noch größeren Vorfallsbereich angewandt, zu dem z. B. auch verbale sexuelle Anspielungen, langes oder häufiges Waschen des Penisses des kleinen Sohnes u. a. m. gehören. Offenbar liegen teilweise überzogene Forderungen nach kindlichem Unangetastetbleiben durch wirkliche oder im konkreten Fall auch nur möglicherweise gegebene Erwachsenensexualität vor.

(2) Die Zahlenbehauptungen beruhen auf extremen Annahmen über die Dunkelziffer. Bekannt gewordene und kriminalstatistisch ausgewiesene Fälle werden mindestens verzwanzigfacht, das so erhaltene Ergebnis oft noch kräftig aufgerundet. Nach einer noch nicht veröffentlichten Dunkelfeldstudie des Kriminologischen Forschungsinstituts Niedersachsen ist dagegen von jährlich 82.000 Fällen sexuellen Mißbrauchs von Kindern bis zum Alter von 14 Jahren auszugehen. Eine ähnliche Schätzung nimmt inzwischen auch der deutsche Kinderschutzbund vor.

(3) Überschätzt wird nicht nur das Vorkommen sexuellen Kindesmißbrauchs, sondern auch der Anteil der Inzestfälle hieran. Die gängige These lautet, daß die meisten sexuellen Mißbrauchserfahrungen in Inzestbeziehungen gemacht würden. $\mathrm{Ja}$, häufig wird sexueller Kindesmißbrauch mit Inzest einfach gleichgesetzt - und als Täter werden nur noch Väter vorgeführt. Die Studie des Hannoverschen Kriminologischen Instituts weist jedoch nur zehn Prozent der Täter als biologische Väter und weitere elf Prozent als Stiefväter aus. Die meisten Täter stammen zwar aus dem sozialen Nahbereich des Opfers, nicht jedoch aus der Familie selbst. Auch die Übergriffe der biologischen Väter betreffen zumeist nicht Inzest, wenn dieser im engeren Sinne als Geschlechtsverkehr aufgefaßt wird, sondern andere sexuelle Mißbrauchsformen.

Schon im Buch von Kavemann und Lohstöter findet sich im Kapitel "Kurzer Blick auf Zahlen und Gesetze" die Angabe, daß nur 25,4 Prozent der Täter "Väter, Stiefväter und andere, dieselbe Wohnung bewohnende Männer wie z.B. Freunde der Mutter" seien. Dabei trägt das Buch, das allgemein vom sexuellen Mißbrauch von Mädchen handelt, den suggestiven und zu falschen Annahmen verführenden Titel Väter als Täter.

In ihrem 1992 veröffentlichten Essay Erregte Aufklärung - Kindesmißbrauch: Fakten und Fiktionen vermutet Katharina Rutschky als Motive für die Übertreibungen und Verzerrungen in der Mißbrauchsdebatte, daß Sozialarbeiter auf diesem Wege Nachfrage nach sich selbst und ihren Institutionen schaffen und Feministinnen über das mißbrauchte Mädchen die Frauenfrage lebendig zu halten suchen.

Zur Einschätzung der durch die Mißbrauchsdebatte im Lande erzeugten psychologischen Stimmung zwei Schlaglichter: Im Januar des Jahres 1994 wurde Frau Rutschky zu Beginn eines Kongresses über sexuellen Mißbrauch durch Demonstranten aus der sozialpädagogischen Helferszene beschimpft und tätlich 
angegriffen. Sie wurde gewürgt, erlebte Todesangst und konnte sich nur mit Mühe befreien. Der Kongreß mußte unter Polizeischutz abgehalten werden.

Im März des Jahres 1994 wurde ein 50jähriger Lehrer aus Nordhorn in der Grafschaft Bentheim, dem 187 Fälle sexueller Übergriffe auf junge Menschen vorgeworfen worden waren, freigesprochen und - laut Urteilsbegründung - "in vollem Umfang rehabilitiert". Nach vagem, unbegründeten Anfangsverdacht hatten sich Eltern offenbar in einen Wahn hineingesteigert. Kinder waren so lange und suggestiv befragt worden, bis sie von Übergriffen berichteten.

Offenbar stimmt nach wie vor, daß Inzest selten ist. Eine 'faire' Einschätzung dieser Seltenheit muß auch in Betracht ziehen, daß Inzest, richtete sich Partnerwahl allein nach der Gelegenheit, zu den verbreitetsten sexuellen Begegnungen überhaupt gehören müßte. Offenbar ist hier eine starke Hemmung wirksam, sei sie mehr biologischer oder mehr kultureller Art.

Nicht zuletzt sollte die meines Wissens von keiner Seite bestrittene Tatsache nachdenklich stimmen, daß Inzest zwischen zwei Volljährigen nur einen Bruchteil aller Inzestfälle ausmacht. Die große Überzahl der vom Inzest betroffenen Frauen bzw. Mädchen ist, wegen der vorherrschenden Inzestdyade Vater-junge Tochter, noch sehr jung, im Falle des Geschwisterinzests sind es beide Geschlechter (Maisch 1968; Finkelhor 1980). Bis auf die Pädophilie, die selbstredend nie in Beziehungen zwischen zwei Erwachsenen vorkommt, liegt hier ein bemerkenswerter Unterschied zu allen anderen Formen sexuellen Verhaltens (und Minderheitenverhaltens) zwischen Individuen vor.

Wenn die Kinder herangewachsen sind und die Kraft zur sexuellen Selbstbestimmung haben, wählen sie als Sexualpartner weder Vater noch Mutter oder Geschwister bzw. verstehen es, sich im Falle von (elterlicher) Bedrängung erfolgreich zu verweigern. Die vom Vater-Tochter-Inzest betroffenen minderjährigen Frauen und Mädchen - hier stimmen praktisch alle kriminologischen, sozialpädagogischen und feministischen Studien überein - sind zudem keine Verführerinnen, sondern Opfer physischer und psychischer väterlicher Gewalt.

Was die Seite der Kinder anbetrifft, spricht also nicht nur die generelle Seltenheit des Inzests, sondern auch die Struktur der wenigen real vorkommenden Fälle für die Position Westermarcks. Auf die sexuelle Außenorientierung der Kinder bezog sich auch der Kern seiner Theorie. Doch sollten Eltern nur deshalb nicht in sexuelle Beziehungen zu ihren Kindern treten, weil sie vom Inzestverbot oder aber vom - eventuell vorweggenommenen - Widerwillen ihrer Kinder zurückgehalten werden? Vorstellbar, wenn nicht sogar wahrscheinlich ist, daß auch bei den Eltern eine mit Kindheit - hier: der ihrer Kinder, die bei ihnen aufwuchsen - verbundene und der Tendenz nach auf sexuelles Desinteresse hinauslaufende Vertrautheit eine Rolle spielt. Wie dem auch sei, eine nur auf einer Seite vorhandene Abneigung reicht als Inzestverhinderung aus, solange diese Haltung von der anderen Seite respektiert wird. 


\section{Inzestbarrieren im Tierreich und ihr biologischer Sinn}

Schon Westermarck selbst hatte auf Inzestbarrieren im Tierreich spekuliert und war sich bewußt, daß seine Theorie mit häufigen Paarungen zwischen nahe verwandten Individuen bei Tieren unvereinbar wäre. Nach Forschungen aus den letzten zwei bis drei Jahrzehnten kann als gesichert gelten, daß Inzest bei fast allen sexuell sich fortpflanzenden Tierarten selten ist, wobei unterschiedliche Meidungsmechanismen wirksam sein können (siehe Überblick und Einteilung bei Bischof 1972; 1973; 1985, 261-290, 310-324, 348-368; speziell zur Inzestvermeidung nichtmenschlicher Primaten: Tokuda 1961; Imanishi 1965; Kaufmann 1965; Sade 1968; Itani 1972; Demarest 1977; Packer 1979; Pusey 1980). Parallelen zur Inzestvermeidung bei Tieren existieren darüber hinaus auch im Pflanzenreich (Fitter 1987, $260 \mathrm{ff}$.)

Eine größere Inzestwahrscheinlichkeit dürfte sowieso nur bei solchen Tierarten zu erwarten sein, bei denen ein oder beide Elternteile mit ihrem Nachwuchs in Familien zusammenleben. Hier kommt es jedoch entweder zur Familienauflösung noch vor der Geschlechtsreife der Jungen oder, bei fortbestehendem Familienverband, zu Phänomenen innerfamiliärer Sexualhemmung.

Familienauflösungen geschehen zumeist durch Abwanderung oder Vertreibung der Jungtiere, die sich in der Folgezeit so stark zerstreuen, daß künftige Inzestchancen, wie bei nicht-familienbildenden Arten, nur minimal gegeben sind. Auch wenn, wie es bei vielen in Mutterfamilien sich organisierenden Säugetierarten der Fall ist, nur das männliche Geschlecht abwandert, ist hiermit dem Inzest vorgebeugt. Bei manchen haremhaltenden Säugetierarten, bei denen die weiblichen Jungtiere im Gegensatz zu den männlichen weder aus eigenem Impuls fortziehen noch vertrieben werden, kommt es zu ihrer 'Entführung' durch fremde Männchen. Bei anderen dieser Arten wird die Vater-Tochter-Verpaarung durch den rechtzeitigen Wechsel des Haremhalters verhindert.

Bei einigen Säugetierarten bleiben erwachsene Jungtiere noch längere Zeit im erweiterten Familienverband, wobei sich jedoch allein die Eltern - das sogenannte Alpha-Paar - weiter fortpflanzen dürfen. Die sexuelle Inaktivität des herangewachsenen Nachwuchses kann über Drohung und ggf. Intervention (eines) der Eltern oder anderer rangüberlegener Tiere der Gruppe sichergestellt werden. Häufig ist sie jedoch bei den betreffenden Arten psychosomatisch bedingt ('psychische Kastration').

Bei verschiedenen in größeren Gruppen lebenden Primatenarten, u.a. beim Schimpansen, kann im Falle fortdauernder Mutter-Sohn- und Geschwisterkontakte gegenseitiges sexuelles Desinteresse beobachtet werden. Da die Tiere ansonsten sexuell aktiv sind, ist dies vermutlich der Ausfluß der langen Vertrautheit in den Beziehungen.

Im Falle der Familienauflösung und räumlichen Verteilung ist nur eine geringe Inzestwahrscheinlichkeit gegeben, jedoch kein spezieller Mechanismus, der bei möglichen Begegnungen nahe verwandter adulter Tiere eine Paarung hindern würde. Im Falle des bei nichtmenschlichen Primaten beobachtbaren Westermarck-Effekts beruht die Hemmung auf dem gemeinsamen Aufwachsen und der 
Erinnerung der Tiere an ihre Kindheitskumpanen. Ein sicheres Verwandtenerkennen ist auch hier nicht gewährleistet. Wo z. B. Väter der Familie nicht angehören, wäre die Vater-Tochter-Dyade nicht gehemmt.

Es wird jedoch noch von weiteren Meidungsmechanismen berichtet. So sollen Wachteln ein andersgeschlechtliches Geschwister als Paarungspartner auch dann meiden, wenn es vor dem Schlüpfen aus dem Nest genommen und in einem anderen Nest aufgezogen wurde. Vermutlich findet hier ein 'Phänotypvergleich' statt, das fremde Geschwister wird als den bekannten Geschwistern physisch ähnlich erkannt (Holmes/Sherman 1987; Gould/Gould 1990). Eine bemerkenswerte Entdeckung in diesem Zusammenhang war, daß Nagetiere genetische Verwandtschaft geruchlich und damit unabhängig von früher Assoziation erkennen und auf diese Weise geeignete Paarungspartner 'herausriechen' können (Beauchamp/Yamazaki/ Boyse 1988; Gould/Gould 1990).

Bei mehreren der aufgelisteten Verhaltensformen ist es fraglich, ob sie wegen ihrer inzestverhindernden Wirkung auch evoluiert sind. Offensichtlich scheinen sie noch andere Funktionen zu erfüllen wie das Sammeln von Erfahrung, die Gewinnung eigener Territorien oder das Fernhalten von Sexualkonkurrenten. Inzestvermeidung könnte dann eine ihrer Funktionen, in manchen Fällen aber auch nur einen Nebeneffekt darstellen. Die Allgemeinheit der Inzestvermeidung im Tierreich dürfte aber wohl kaum als Zufall anzusprechen sein.

Dies wird vor allem dann deutlich, wenn man bedenkt, daß Fortpflanzung zwischen nahen Verwandten auch gewichtige Vorteile in sich bergen würde. Bei einem solchen Fortpflanzungsmodus wäre eine besonders risikolose, zeit- und energiesparende Partnerfindung gewährleistet. Einmal erprobte und bewährte genetische Kombinationen würden nicht mehr zugunsten anderer unerprobter Möglichkeiten aufgebrochen. Darüber hinaus würden aufgrund der teilweisen, im Falle fortgesetzter enger Inzucht schließlich nahezu vollständigen genetischen Übereinstimmung der Eltern besonders viele Kopien eigener Gene an den Nachwuchs weitergegeben. Dem in der Natur herrschenden Prinzip der Genkonkurrenz wäre optimal Rechnung getragen.

Fortpflanzung zwischen nahen Verwandten müßte also eigentlich in der Natur weit verbreitet sein - wenn mit ihr nicht auch gravierende Nachteile verbunden wären, die die angesprochenen Vorteile sogar überwiegen und letztlich eine Negativbilanz schaffen.

In der Diskussion über die evolutiven Nachteile der Inzucht gibt es zwei Auffassungen. Nach der ersten besteht das Problem in dem durch Inzucht bewirkten Manifestwerden von zuvor 'unsichtbaren' Erbschäden. Dies beruht auf der mit Inzucht einhergehenden vermehrten Homozygotenbildung (d.h. der Zunahme von mit gleichartigen Erbanlagen besetzten Genorten) und damit auch dem Homozygotwerden regelmäßig vorhandener schädlicher rezessiver Gene. Die Wahrscheinlichkeit der Produktion lebensunfähigen und kranken Nachwuchses hängt hierbei stark vom Verwandtschaftsgrad der inzüchtenden Eltern ab und ist bei inzestuöser Fortpflanzung, als extremster Form von Inzucht, mit Abstand am höchsten. 
Eine Vielzahl von Tierbefunden, aber auch die wenigen über Einzelfallstudien hinausgehenden Untersuchungen an menschlichen Inzestkindern (Carter 1967; Adams/Neel 1967; Seemanova 1971; Baird/McGillivray 1982) zeigen deutlich die große Gefahr, die der Fortpflanzung zwischen nächsten Verwandten innewohnt. Andererseits demonstrieren Tierzüchtungsversuche den auch theoretisch zu erwartenden Effekt, daß nach lange genug fortgesetzter enger Inzucht schließlich ein Rückgang in der Belastung mit Erbschäden eintritt, bis hin zur Enstehung einer besonders erbgesunden Population (Livingstone 1969). Das heißt, daß entweder in freier Natur für Inzuchtpopulationen kaum eine Chance besteht, jene Gesundungsphase zu erreichen - etwa aufgrund der Konkurrenz mit nichtinzüchtenden Populationen -, oder aber, daß der biologische Sinn der Inzestvermeidung letztlich mit einer Verhinderung von Erbschäden gar nicht im Zusammenhang steht.

Letzteres behauptet die andere hier bestehende Hypothese. Sie verknüpft den Vorteil der Inzestvermeidung mit dem Zweck der zweielterlichen sexuellen Fortpflanzung im allgemeinen. Die sexuelle Reproduktion diene der Schaffung einer Vielfalt genotypischer Varianten als Reserve für den Fall des Eintretens neuartiger Umweltbedingungen. Durch fortgesetzte enge Inzucht wird jedoch die genetische Vielfalt wieder aufgehoben, entsteht, genetisch betrachtet, eine Situation, die sich von der ungeschlechtlichen Fortpflanzung, die in der Natur ebenfalls vorkommt und die ursprüngliche Vermehrungsmethode darstellt, kaum noch unterscheidet. Der biologische Sinn der Inzestvermeidung wäre somit derselbe wie der von Sexualität im allgemeinen (Bischof 1985, 391-413).

Die Diskussion um die Überlegenheit der sexuellen Fortpflanzung ist zur Zeit im vollen Gange (für einen Überblick siehe Gould/Gould 1990; LeVay 1994), so daß die zuletzt vorgestellte These möglicherweise modifiziert werden muß.

\section{Das Verbot}

Warum existiert beim Menschen ein gesellschaftliches Inzestverbot, wenn dem Inzest doch schon auf natürlicher Basis vorgebeugt ist? Es wurde bereits darauf hingewiesen, daß Instinkte nicht unfehlbar sein müssen, so daß sich das Verbot gegen wenige Außenseiter richten könnte. Doch warum läßt man sie nicht gewähren? Zumal es recht fraglich ist, ob durch das Verbot überhaupt eine irgendwie nennenswerte Anzahl von Inzestfällen verhindert wird. Denn auch bei seiner Abwesenheit geschähe Inzest vermutlich nur selten, und in den Bereich der Sexualität eingreifenden Normen ist generell eher nur geringer Erfolg beschieden.

Das Inzestverbot überschneidet sich außerdem mit verschiedenen anderen Normen in unserer Kultur, vor allem mit den Normen zum Schutz der sexuellen Selbstbestimmung. Der Kern des Inzestverbots müßte aber doch wohl solche sexuellen Vorkommnisse betreffen, die ansonsten nicht verboten sind. Nach Gesetzeslage der BRD wäre dies der (beidseitig) freiwillig vollzogene Geschlechtsverkehr zwischen Eltern und volljährigen Kindern und zwischen volljährigen Geschwistern. 
Zweifelhaft ist, ob das Verbot aus erbhygienischen Gründen existiert und damit nicht nur dieselbe Wirkrichtung besitzt wie die natürliche Inzestvermeidung, sondern auch ihren biologischen Sinn nachzeichnet. Ein starkes Argument ist hier, daß die Reaktionen der Abscheu und Irritation, die mit dem Bekanntwerden realer Inzestfälle oder auch nur der Vorstellung von Inzest einhergehen, wohl kaum befriedigend mit der Sorge um erbkranke Kinder erklärt werden können. Den eingangs dargestellten traditionellen soziologisch-ethnologischen Thesen zur Funktion des Verbots kommt wiederum nur wenig Überzeugungskraft zu, da sie auf der falschen Prämisse vom starken Inzestwunsch des Menschen fußen (Klein 1991, 156-180).

Ein maßgeblicher Grund für das Verbot dürfte vielmehr darin liegen, daß durch die wenigen Außenseiter, die Inzest praktizieren, Verhaltenserwartungen gestört werden, die an der natürlichen Inzestscheu orientiert sind. $\mathrm{Da}$ an diese Verhaltenserwartungen auch Sinnorientierungen geknüpft sind und der Mensch einem allgemeinen Konformitätsbedürfnis unterliegt, wird stattfindender Inzest als bedrohlich erlebt. Zum zweiten spielt eine Rolle, daß beim unwillkürlichen Versuch, sich in reale Inzesttäter hineinzuversetzen, Irritation und Abscheu aufkommen, weil man selbst der natürlichen Inzestscheu unterliegt (Bischof 1985, 567ff.; Klein 1991, 185-192).

Nach aufgeklärtem demokratischen Rechtsverständnis läßt sich ein Verbot auf einer solchen Grundlage jedoch nicht legitimieren. Personen, die Inzest praktizieren und deren Verhalten bekannt wird, werden zwar als irritierend und bedrohlich erlebt, doch unternehmen sie, die Freiwilligkeit des Inzests vorausgesetzt, keinen Übergriff auf die Belange und Interessen anderer.

\section{Bibliographie}

Aberle, D. F. et al. (1963), The Incest Taboo and the Mating Patterns of Animals, in: American Anthropologist 65, 253-265

Adams, M. S. /J. V. Neel (1967), Children of Incest, in: Pediatrics 40, 50-62

Armstrong, L. (1978), Kiss Daddy Good-Night, New York

Baird, P. A./B. McGillivray (1982), Children of Incest, in: The Joumal of Pediatrics 101, 854-857

Beauchamp, G. K./K. Yamazaki/E. A. Boyse (1988), Geruchliche Ausprägung von genetischen Unterschieden, in: Biologie des Sozialverhaltens. Spektrum der Wissenschaft: Verständliche Forschung, Heidelberg, 160-168

Bischof, N. (1972), Inzuchtbarrieren in Säugetiersozietäten, in: Homo 23, 330-351

- (1973), Die biologischen Grundlagen des Inzesttabus, in: G. Reinert (Hrsg.), Bericht über den 27. Kongreß der Deutschen Gesellschaft für Psychologie in Kiel 1970, Göttingen, 115-142

- (1985), Das Rätsel Ödipus, München

Carter, C. O. (1967), Risk to Offspring of Incest, in: Lancet 1, 436 
Demarest, W. J. (1977), Incest Avoidance among Human and Nonhuman Primates, in: S. Chevalier-Skolnikoff/F. E. Pourier (eds.), Primate Biosocial Development, New YorkLondon, 323-342

Ellis, H. (1906), Sexual Selection in Man, Philadelphia

Finkelhor, D. (1980), Sex among Siblings, in: Archives of Sexual Behavior 9, 171-194

Fitter, A. (1987), Blumen. Wildbliuhende Pflanzen, Hamburg-Berlin

Fortune, R. F. (1932), Incest, in: E. R. A. Seligman (ed.), Encyclopeadia of the Social Sciences 7, New York, 620-622

Fox, R. (1962), Sibling Incest, in: British Journal of Sociology 13, 128-150

Frazer, Sir J. G. (1910), Totemism and Exogamy. 4. Bd, London

Freud, S. (1972), Die Traumdeutung, Studienausgabe Bd. 2, Frankfurt/M (zuerst 1900)

- (1974), Totem und Tabu, Studienausgabe Bd. 9, Frankfurt/M (zuerst 1912/13)

Gardiner Sirtl, A. (Hrsg.) (1983), Als Kind mißbraucht. Frauen brechen das Schweigen, München

Gould, J. L./C. G. Gould (1990), Partnerwahl im Tierreich, Heidelberg

Harris, M. (1980), Culture, People, Nature, New York

Holmes, W. G./P. W. Sherman (1987), Verwandtenerkennen bei Tieren, in: K. R. Scherer/A. Stahnke/P. Winkler (Hrsg.), Psychobiologie. Wegweisende Texte der Verhaltensforschung von Darwin bis zur Gegenwart, München, 403-414

Imanishi, K. (1965), The Origin of the Human Family: A Primatological Approach, in: S. A. Altman/K. Imanishi (eds.), Japanese Monkeys: A Collection of Translations, Alberta, 113-140

Itani, J. (1972), A Preliminary Essay on the Relationship between Social Organization and Incest Avoidance in Nonhuman Primates, in: Primate Sozialisation, New York, 165171

Justice B./R. Justice (1979), The Broken Taboo, New York

Kaufmann, J. H. (1965), A Three-Year Study of Mating Behavior in a free Ranging Band of Rhesus Monkeys, in: Ecology 46, 500-512

Kavemann, B./. Lohstöter (1984), Väter als Täter, Hamburg

Klein, J. (1991), Inzest: Kulturelles Verbot und naturliche Scheu, Opladen

Lappessen, K. (1991), Was ist mit Anna, München

Leavitt, G. C. (1990), Sociobiological Explanations of Incest Avoidance: A Critical Review of Evidential Claims, in: American Anthropologist 92, 971-993

LeVay, S. (1994), Keimzellen der Lust. Die Natur der menschlichen Sexualität, Heidelberg

Lévi-Strauss, C. (1984), Die elementaren Strukturen der Verwandtschaft, Frankfurt/M (zuerst 1949: Les structures elementaires de la parenté)

Livingstone, F. B. (1969), Genetics, Ecology and the Origins of Incest and Exogamy, in: Current Anthroplogy 10, 45-61

Maisch, H. (1968), Inzest, Reinbek

Malinowski, B. (1931), Culture, in: E. R. A. Seligman (ed.), Encyclopedia of Social Sciences. Bd. 4, London, 621-646

- (1979), Das Geschlechtsleben der Wilden in Nordwest-Melanesien, Frankfurt/M (zuerst 1929: The Sexual Life of Savages in North-Western Melanesia)

- (1981), Geschlecht und Verdrängung in primitiven Gesellschaften, Frankfurt/M (zuerst 1927: Sex und Repression in Savage Society) 
McCabe, J. (1983), FBD Marriage: Further Support of the Westermarck Hypothesis of the Incest Taboo?, in: American Anthropologist 85, 50-69

Murdock, G. P. (1949), Social Structure, Toronto

Packer, C. (1979), Inter-troop Transfer and Inbreeding Avoidance in PapioAnubis, in: Animal Behaviour 27, 1-36

Parsons, T. (1954), The Incest Taboo in Relation to the Social Structure and the Socialization of the Child, in: British Journal of Sociology 5, 101-107

Pusey, A. E. (1980), Inbreeding Avoidance in Chimpanzees, in: Animal Behaviour 28, 543552

Rijnaarts, J. (1988), Lots Töchter. Über den Vater-Tochter-Inzest, Düsseldorf

Rush, F. (1982), Das bestgehiltete Geheimnis, Berlin

Rutschky, K. (1992), Erregte Auklärung. Kindesmißbrauch: Fakten und Fiktionen, Hamburg

Sade, D. S. (1968), Inhibition of Son-Mother Mating among Free-Ranging Rhesus Monkeys, in: J. H. Messerman (ed.), Science and Psychoanalysis. Bd. 12: Animal and Human, New York, 18-38

Seemanova, E. (1971), A Study of Children of Incestuous Matings, in: Human Heredity 21, 108-128

Seligman, B. Z. (1929), Incest and Descent: Their Influence on Social Organization, in: Journal of the Royal Anthropological Institute 59, 231-272

- (1932), The Incest Barrier: Its Role in Social Organization, in: British Journal of Psychology 22, 250-276

- (1935), The Incest Taboo as a Social Regulation, in: The Sociological Review 27, 75-93

- (1950), The Problem of Incest and Exogamy: A Restatement, in: American Anthropologist 52, 305-316

Shepher, J. (1971), Mate Selection among Second-Generation Kibbutz Adolescents and Adults. Incest Avoidance and Negative Imprinting, in: Archives of Sexual Behavior 1, 293-307

- (1983), Incest - A Biosocial View, New York-London

Spiro, M. E. (1958), Children of the Kibbutz, Cambridge MA

Talmon, G. Y. (1964), Mate Selection in Collective Settlements, in: American Social Review 29, 408-491

Tokuda, K. (1961), A Study of Sexual Behaviour of the Japanese Monkey Troop, in: Primates 3, 1-41

White, L. A. (1948), The Definition and Prohibition of Incest, in: American Anthropologist 50, 416-435

Westermarck, E. (1894), The History of Human Marriage, London (zuerst 1891)

- (1921), The History of Human Marriage (5. erweiterte Ausgabe), London

- (1934), Recent Theories of Exogamy, in: Three Essays on Sex and Marriage, London, 127-159

Wolf, A. P. (1966), Childhood Association, Sexual Attraction and the Incest Taboo: A Chinese Case, in: American Anthropologist 68, 883-898

- (1968), Adopt a Daughter-in-Law, Marry a Sister: A Chinese Solution of the Problem of the Incest Taboo, in: American Anthropologist 70, 864-874 
- (1970), Childhood Association and Sexual Attraction. A Further Test of the Westermarck Hypothesis, in: American Anthropologist 72, 503-515

Wolf, A. P./C. Huang (1980), Marriage and Adoption in China 1845-1945, Stanford

Zimmer, D. E. (1984), Wenn wir schlafen und träumen. Die Nachtseite unseres Lebens, München

- (1986), Tiefenschwindel. Die endlose und die beendbare Psychoanalyse, Reinbek 